\title{
Il superleader ("ordinario") Matteo Salvini e il suo corpo
}

\author{
Marco Mazzoni \\ Dipartimento di Scienze Politiche, Università degli Studi di Perugia \\ marco.mazzoni@unipg.it \\ Roberto Mincigrucci \\ Dipartimento di Scienze Politiche, Università degli Studi di Perugia \\ roberto.mincigrucci@unipg.it
}

\begin{abstract}
According to scholars, today's political leaders face with the "paradox of democratic leadership": on the one hand, they have to appear as strong statesmen, in possession of particular skills that gave them the authority to rule the people. On the other hand, they are allowed to rule by the people, and they have to appear similar and intimate with common people. Analyzing the pictures that Salvini publishes on his personal Facebook profile, we try to understand how Matteo Salvini aims to solve the aforementioned paradox. Our main hypothesis is that Salvini implements a strategic use of his "political body", mixing the features of a "super leader" with the ones of an "ordinary person”.
\end{abstract}

\section{Keywords}

Salvini; Facebook; Celebrity politics; Leadership; Visual content analysis

\section{Sommario/Content}

1. Introduzione

2. Il paradosso del leader democratico

3. Metodologia

4. Il super celebrity politician: Salvini il "capitano"

5. L'everyday celebrity politicians - Salvini "l'ordinario"

6.Conclusioni

Bibliografia 


\section{Ocula ${ }^{22}$}

Vol 21, No 22 (April 2020) • DOI: 10.12977/ocula2020-10

Marco Mazzoni e Roberto Mincigrucci | Il superleader ("ordinario"). Matteo Salvini

e il suo corpo

\section{Introduzione}

Nel 2008 Federico Boni pubblicava il libro Il superleader. Fenomenologia mediatica di Silvio Berlusconi, un'analisi che riguardava «non tanto la spettacolarizzazione della politica, ma la spettacolarizzazione di Berlusconi, e la sua mediatizzazione» (Boni 2008: 9). Il lavoro di Boni è un viaggio all'interno di uno dei fenomeni politici e mediatici più interessanti che il nostro paese abbia mai conosciuto, indagando le condizioni di produzione e riproduzione di un corpo, quello appunto di Berlusconi, diventato paradigmatico delle più rilevanti trasformazioni dello scenario politico e mediatico italiano. A distanza di dieci anni Berlusconi appare sempre meno "super", a causa probabilmente dell'età che avanza che gli impedisce di cimentarsi in contesti variegati come agli inizi della sua carriera politica, ma soprattutto è sempre meno "leader", con le elezioni del 2018 che hanno certificato una crisi di consensi della propria compagine politica e l' incapacità di tenere le redini di quella che era la coalizione di centro destra.

$\mathrm{Al}$ suo posto si è affermato però un nuovo "superleader", ovvero Matteo Salvini, capace di portare la nuova "Lega Nazionale" (Vampa 2018: 57) dal 4 per cento dei consensi del 2013 al 34,3 per cento delle elezioni europee 2019, e che ancora oggi registra percentuali di gradimento molto elevate secondo la maggior parte dei sondaggi di opinione. Il superleader Berlusconi veniva descritto da Boni come un personaggio onnipresente nel panorama mediatico, oggetto d'attenzione spasmodica, raccontato in diversi contesti e tramite diversi frame narrativi. Lo stesso si può dire a proposito di Salvini, seppur con modalità diverse e con messaggi diversi. Come Berlusconi dieci anni fa, anche Salvini oggi è al centro del dibatto pubblico. Non soltanto del dibattito politico, ma anche delle notizie di carattere più soft, come i suoi interventi nei programmi di intrattenimento o il suo coverage nei giornali di gossip dimostrano. Inoltre, come Berlusconi, anche Salvini fa del corpo un elemento centrale della sua comunicazione, rendendolo una vera e propria ideologia iconica e linguistica. Questo rende Salvini una vera e propria "icona pop", ovvero un personaggio emblematico di un'epoca e di una cultura, nello specifico della cultura popolare contemporanea. Secondo Spaziante (2016), le icone pop sono dei personaggi, entità reali che al contempo appartengono all'immaginario collettivo e la cui effige diviene visivamente rappresentativa di un determinato periodo storico, di un particolare stile o di una particolare cultura. Tali personaggi sono delle figure abili a costruire attorno a loro una narrazione e una comunicazione, in un contesto in cui la vita privata $\mathrm{e}$, più in generale, alcuni spazi che Meyerowitz (1985) definirebbe del "retroscena", diventano determinanti per costruire una identità narrativa facilmente accessibile ad un ampio pubblico di fruitori. Come vedremo, Salvini è un personaggio glamour, capace di fare notizia non soltanto per i suoi provvedimenti politici, ma anche per i suoi comportamenti e per il suo modo di vestire. I suoi elettori, più che dei militanti o dei sostenitori, possono essere considerati a tutti gli effetti dei "fan" (Diamanti 2019: 16): esaltano il "capitano" più che le proposte politiche, parlano con entusiasmo dei suoi tratti caratteriali e umani, esattamente come avviene per una popstar. 


\section{Ocula}

Vol 21, No 22 (April 2020) • DOI: 10.12977/ocula2020-10

Marco Mazzoni e Roberto Mincigrucci | Il superleader (“ordinario”). Matteo Salvini

e il suo corpo

Lo stesso Spaziante (ivi: 4) sostiene che "un ruolo determinante nel definire l'identità di un'icona pop lo gioca l'immagine, e di conseguenza le scelte che riguardano l'apparenza visiva". Per questo motivo la nostra analisi mira ad indagare, dal punto di vista "visuale", l'uso che Salvini fa del proprio corpo, per entrare in sintonia con la cultura popolare e creare empatia con i suoi elettori. Il tipo di corpo su cui ci focalizzeremo è il "corpo mediale del leader politico" (Boni 2002), inteso come un "costrutto sociale, ovvero un corpo coprodotto potenzialmente da un intergioco di formazioni sociali - in tutte sue le dimensioni, articolazioni e processi" (Pozzi 1994: 129) - e le cui condizioni di produzione e significazione sono determinate dalla sua rappresentazione mediale (Boni 2002: 41).

Quanto conta il corpo nella comunicazione del leader leghista? Che tipo di messaggio veicola attraverso le immagini, le foto e i selfie che vengono diffusi attraverso Facebook? Queste sono le domande che animano la nostra ricerca, partendo dal presupposto che nella comunicazione salviniana nulla è lasciato al caso, perché determinante nella definizione della rappresentazione della sua leadership politica. In particolare, cercheremo di mettere in luce l'abilità del leader leghista di costruire la propria immagine anche attraverso gli atteggiamenti e persino attraverso l'abbigliamento. La rappresentazione sociale del corpo del leader è costituita dall'intreccio di due dimensioni: le immagini mentali e le icone corporee (Pozzi 1994). Le prime riguardano le "rappresentazioni mentali" del corpo, e di alcuni suoi aspetti o funzioni. Le seconde sono invece le rappresentazioni corporee concrete, esplicite e 'tangibili', diffuse all'interno di una (micro)formazione, di un sistema sociale o di un universo culturale (ivi: 127). La nostra ipotesi è che Salvini, grazie ad un sapiente uso delle icone corporee, offre alla propria comunità politica un punto di riferimento comune. Ne interpreta l'identità, l'immagine e il linguaggio. Il leader leghista utilizza i vestiti e gli outfit per costruire delle icone corporee che cambiano a seconda delle stagioni politiche. Salvini ha dimostrato una grande capacità di adattarsi a diverse fasce di pubblico, a seconda delle esigenze comunicative del momento (cfr. Barile e Vagni 2019: 67). Dalla felpa con cui ha dato il via alla scalata della Lega, alla maglietta da poliziotto con cui la sera va a correre per le strade di Roma, per arrivare al torso nudo, divisa "ufficiale" dell'estate, come era già accaduto, e forse non è un caso, a dicembre del 2014 quando nella copertina di Oggi è stato ritratto con addosso solo una cravatta verde. Ovunque il leader leghista si è lasciato immortalare senza maglietta, e questo è un fenomeno del tutto inusuale per una persona che ricopriva la carica di Ministro, ${ }^{1}$ ma forse non insensato. Il consenso politico oggi si basa su valori ed immagini che si identificano in aspetti non politici della vita pubblica e privata (cfr. Delli Carpini e Williams 2001). Lo stesso Spaziante sostiene che per le "icone pop" la vita quotidiana diventa una forma di rappresentazione perenne, «un palco quotidiano per una vita messa in scena» (ivi: 18). Dopotutto, già in studi più datati, Meyrowitz (1985) suggeriva che l'elevata esposizione mediatica dei

1 La nostra analisi si basa su un periodo di tempo in cui Salvini ricopriva la carica di Ministro dell'Interno e Vicepremier. 


\section{Ocula}

Vol 21, No 22 (April 2020) • DOI: 10.12977/ocula2020-10

Marco Mazzoni e Roberto Mincigrucci | Il superleader (“ordinario”). Matteo Salvini

e il suo corpo

leader avrebbe comportato una "perdita dell'aura" o una "desacralizzazione" della figura del leader politico, derivante soprattutto dalla "messa in scena" di quello che prima dell'avvento dei media elettronici era considerato "il retroscena". Quello che cercheremo di mettere in evidenza nel corso dell'analisi è che tale "perdita dell'aura" non è un fenomeno prettamente subito dal leader in questione, ma in alcune situazioni diventa una scelta ragionata al fine di "avvicinarsi" ed "identificarsi" con la propria comunità di elettori. Diversi autori (v. Achen e Bartels 2016; Green, Palmquist e Schickler 2004) sostengono che negli ultimi tempi il voto si orienta più su basi identitarie che sulle ideologie o sui programmi politici. In altre parole, un politico non deve essere solo il fautore di una determinata proposta politica, ma deve incarnare, con i suoi comportamenti e con il suo modo di essere, la proposta stessa, deve essere coerente con il gruppo sociale che intende rappresentare (cfr. Kreiss 2018), con una piena adesione al modello di vita che vuole identificare (cfr. Mancini 2011). Il corpo diviene un'ideologia iconica e linguistica, perché permette al superleader di poter dire alla sua comunità: sono come voi, anzi uno di voi.

Il presente lavoro si basa su un'analisi delle immagini "postate" nella pagina Facebook di Matteo Salvini. L'utilizzo delle immagini sta diventando sempre più rilevante nelle strategie comunicative dei politici (v. Karadimitriou e Veneti 2016) in quanto, rispetto ai messaggi testuali e alle dichiarazioni, sono strumenti più immediati, facilmente memorizzabili, e in grado di attrarre l'attenzione del pubblico (cfr. Muñoz e Towner 2017: 3), anche di quello meno attento alle vicende politiche. Si è deciso di analizzare Facebook in quanto, da una parte è il social network preferito da Salvini per veicolare i propri messaggi (cfr. Carone e Cavallaro 2019: 51), ma soprattutto è il social che più si presta ai nostri obiettivi di ricerca. La "pagina pubblica" infatti può essere considerata lo strumento ideale in un contesto di campagna permanente (cfr. Ceccobelli 2017) perché permette di pubblicare con un certo grado di discrezionalità i propri messaggi e le proprie immagini senza nessun tipo di filtro o mediazione, in un ambiente mediale nel quale i "post" possono raggiungere percentuali non irrisorie di elettori. L'utilizzo intensivo di tale strumento sottintende anche delle scelte strategiche sul messaggio che il leader intende veicolare e che contribuiscono a rafforzare il suo status di incona pop, perfettamente integrata con il contesto culturale a cui si rivolge. Come spiegano Barile e Vagni in un recente saggio (2019), la nuova egemonia culturale imposta dagli influencer sta smantellando le routine produttive e l'autorità del giornalismo tradizionale. L'immagine pura e ad alta definizione che caratterizzava la comunicazione politica negli anni '8o e '9o sta lasciando il passo a delle immagini a bassa risoluzione e talvolta amatoriali (o presunte tali). Questo tipo di immagini, definite "lo-fi" (ivi: 64), ovvero a bassa fedeltà, sono di qualità inferiore rispetto a quelle prodotte da dei mezzi elettronici sempre più sofisticati e che potevano contare sulla competenza di veri professionisti, tuttavia contribuiscono a veicolare l'immagine di un leader più imperfetto e quindi più "vero", "autentico" e comune. L'utilizzo di una strategia di comunicazione lo- $f$ quindi diventa centrale nel rafforzare lo status di icona pop del leader della Lega, in quanto contribuisce a condurre le sue modalità espressive su un piano collo- 


\section{Ocula}

Vol 21, No 22 (April 2020) • DOI: 10.12977/ocula2020-10

Marco Mazzoni e Roberto Mincigrucci | Il superleader (“ordinario”). Matteo Salvini

e il suo corpo

quiale e gli permette di stabilire con i suoi follower un'affiliazione più spontanea e duratura. In altre parole, i social network come Facebook consentono ai politici di parlare direttamente al "popolo", bypassando i media mainstream (v. Bartlett 2014; Engesser, Fawzi, Larsson 2017) e veicolando un'immagine più "normale" e perfettamente integrata con la cultura popolare: una caratteristica questa che li rende particolarmente appetibili per i leader populisti (cfr. Kriesi 2014: 367). Non a caso, Salvini è il leader italiano più attivo sulla piattaforma di Zuckerberg (v. Bobba 2019).

\section{Il paradosso del leader democratico}

In un recente articolo, De Vreese e altri autori (2018) suggeriscono di affrontare il populismo come un "communication phenomenon", ovvero di non soffermarsi soltanto sui tratti distintivi della cosiddetta "ideologia populista" (Mudde 2004: 543), ma anche nei modi in cui questa viene comunicata. Secondo gli autori, il contenuto del messaggio, ovvero la divulgazione delle componenti principali dell'ideologia populista, insieme allo stile comunicativo, sono elementi chiave del fenomeno populista e Salvini ha dimostrato (e sta dimostrando) di essere un abile comunicatore.

Come ricorda Fabio Turato (2018) nel suo ultimo libro, quando Salvini ha iniziato la sua scalata alla segreteria del partito, la Lega era ai suoi minimi storici, reduce da dei risultati elettorali tutt'altro che entusiasmanti, ma soprattutto con la base in rivolta a causa degli scandali che avevano coinvolto la famiglia Bossi e altri personaggi apicali del partito. Al fine di ridare entusiasmo ai suoi militanti e di riconquistare la fiducia degli elettori, Salvini ha cambiato radicalmente lo schema di comunicazione della Lega (cfr. Turato 2018). Innanzitutto è intervenuto sullo "stile", cercando un rapporto più diretto con il suo "popolo", attraverso anche un utilizzo massiccio dei social network. Inoltre, ha cambiato anche il "contenuto", mettendo da parte le istanze federaliste, autonomiste e a tratti secessioniste (cfr. Passarelli e Tuorto 2018: 268) e ha accentuato le posizioni antisistema che già erano presenti nel partito, affrontando tematiche come gli effetti della globalizzazione, il ruolo dei confini, l'identità del popolo e la sfiducia nei confronti delle élite e del potere (v. Turato 2018; Bobba 2019). In altre parole, Salvini si è schierato dalla parte dei "perdenti della globalizzazione" (Kriesi et al. 2006: 922), sposando appieno l'ideologia populista che contrappone il "popolo buono" contro le "élite corrotte" (Mudde 2004: 543).

Questo tipo di narrazione però pone Salvini di fronte a quello che Kane e Patapan (2012) definiscono "the paradox of the democratic leader", ovvero la necessità di apparire in una duplice veste: come uno statista, al di sopra di tutto e di tutti ("above us"), in grado di meritare la fiducia degli elettori per poter governare, ma allo stesso tempo anche una persona comune ("like us"), autentica, con i suoi pregi e difetti, dato che il leader è un cittadino e può rappresentarci perché è come un qualsiasi membro del suo popolo, anzi uno "del popolo". Il leader che si candida a guidare una determinata comunità politica deve produrre un'identificazione con la comunità stessa. Deve quindi costru- 


\section{Ocula}

Vol 21, No 22 (April 2020) • DOI: 10.12977/ocula2020-10

Marco Mazzoni e Roberto Mincigrucci | Il superleader (“ordinario”). Matteo Salvini

e il suo corpo

ire una narrazione che permetta agli elettori di percepirlo come uno di loro. Ma soprattutto deve riuscire a creare il giusto mix tra speciale e ordinario (cfr. Van Zoonen 2005), mostrandosi sia speciale per dimostrare di essere adatto al ruolo di guida sia ordinario e quindi in grado di capire le esigenze dell'elettorato. Come scrive Street in un'interessante analisi su Donald Trump, un punto saliente della comunicazione del presidente americano è l'autenticità: "il fatto che Trump non sembri o non si comporti come un tipico politico gli ha fatto guadagnare punti in termini di autenticità" (Street 2019: 8).

Per tornare alle domande di ricerca, il nostro obiettivo è quello di capire il tipo di uso che Salvini ha fatto del suo corpo, al fine di superare il "paradosso del leader democratico". Lo faremo prendendo spunto dalla distinzione proposta da Wood e altri autori tra "super celebrity politicians" [SCP] e "everyday celebrity politicians" [ECP] (Wood, Corbett, Flinders 2016: 583). La super celebrity politician è quel personaggio "speciale", "eccezionale", "unico", che può contare su un "super talento". Il secondo caso invece riguarda quel leader che vuole apparire "more everyday, more normal, more ordinary" (ivi: 584). Non è un caso che i due autori, nelle due definizioni, usano il termine celebrity: il loro studio parte proprio dal presupposto che il leader politico è a tutti gli effetti una celebrità (v. Marsh, Hart, e Tindall 2010; Street 2004, 2012; Wheeler 2013; Mazzoni e Ciaglia 2015). Come un attore o un cantante, anche il leader politico gode di un'estesa visibilità mediatica che lo rende una persona facilmente riconoscibile anche per il cittadino comune. L'affermarsi del concetto di "celebrità" applicato ai leader politici può essere considerato un tentativo per risolvere il paradosso sopra illustrato: Street (2004) in particolare afferma che la celebrità è un fenomeno potenzialmente importante per rinvigorire la democrazia dato che i politici lo possono usare per connettersi meglio con un pubblico scontento, e questo può essere fatto anche attraverso un uso strategico del proprio corpo (cfr. Boni 2008).

Nell'articolo, Wood et al. (ivi), indagando il contesto inglese, propongono la distinzione tra SCP e ECP per poter rivelare come la grande sfiducia che regna nel campo della politica abbia reso maggiormente necessario per un leader realizzare il secondo tipo di celebrità [ECP], perché mostrando le proprie fragilità, emozioni e imperfezioni il leader appare come una persona normale ("normal human") di cui ci si può fidare. Nel nostro caso invece cercheremo di dimostrare che l'uso che Salvini fa del proprio corpo punta a trovare il giusto mix tra "super eroe" e "persona normale", in modo da poter indossare abiti diversi (la maglia della polizia, la giacca, persino il torso nudo di cui si è parlato prima) a seconda delle situazioni, così da apparire, per dirla con un linguaggio poco accademico, il super eroe di "noialtri”.

\section{Metodologia}

I risultati che verranno presentati nelle prossime pagine sono frutto di un'analisi di tutte le immagini pubblicate nel profilo pubblico di Facebook di Salvini dal 1 luglio al 30 settembre 2018 (ovvero un periodo in cui Salvini ricopriva la carica di Ministro dell'Interno e Vicepremier) tramite un'apposita 


\section{Ocula ${ }^{22}$}

Vol 21, No 22 (April 2020) • DOI: 10.12977/ocula2020-10

Marco Mazzoni e Roberto Mincigrucci | Il superleader ("ordinario"). Matteo Salvini

e il suo corpo

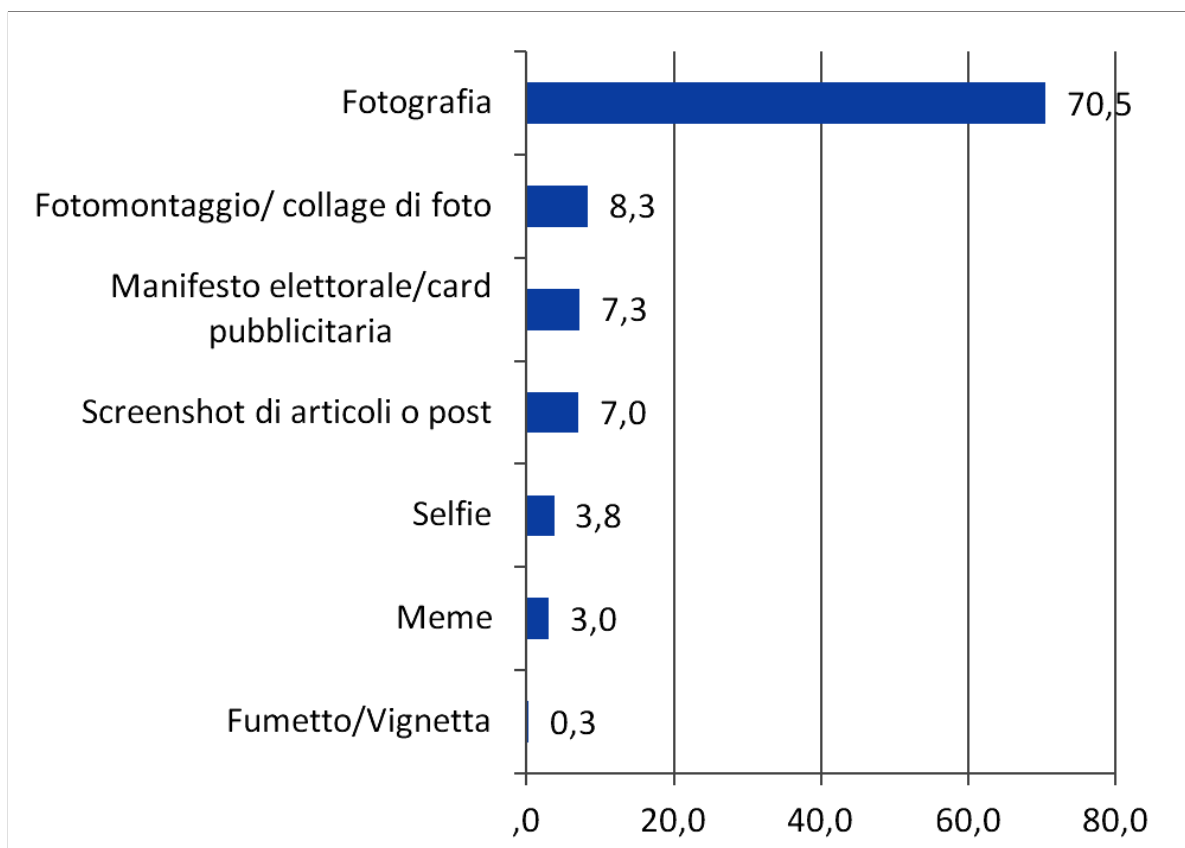

Figura 1. Riepilogo delle immagini analizzate (\%) (Tot. 772).

scheda di analisi. Sono state analizzate tutte le immagini che comparivano nelle sezioni "foto del diario" e "caricamenti dal cellulare". In totale le immagini prese in esame sono state 772. Queste immagini non comprendono soltanto le fotografie vere e proprie, ma delle tipologie di immagini molto variegate. Oltre alle fotografie infatti abbiamo individuato altri sei tipi di immagine: $i$ "meme", ovvero delle raffigurazioni di persone, spesso colte in pose bizzarre o particolari, accompagnate da un messaggio testuale, che hanno lo scopo di fare ironia o, cosa molto comune nel profilo di Salvini, contestare o umiliare un determinato soggetto (cfr. Mazzoleni e Bracciale 2019: 9); fumetti o vignette disegnate; dei manifesti elettorali o delle card pubblicitarie con un breve messaggio testuale volto a pubblicizzare degli eventi o una particolare attività; degli screenshot pubblicati per dare visibilità a post pubblicati sui social network da altri soggetti $\mathrm{o}$ ad articoli di giornale, fotomontaggi o collage di più foto aggregati in una stessa immagine; oppure dei selfie, cioè degli autoritratti scattati dal protagonista della foto. Nella figura 1 sono riportate le varie tipologie di immagini analizzate.

Oltre alla "tipologia di immagini", la scheda di analisi prevedeva l'individuazione di altre variabili costruite sulla base di una letteratura internazionale sull'analisi del contenuto delle immagini (Grabe e Bucy 2009; Schill 2012).

Le variabili che verranno discusse nelle prossime pagine sono:

- Il soggetto principale dell'immagine, che poteva essere una persona fisica, come Salvini stesso, un familiare, un collega oppure un oggetto particolare, come ad esempio del "cibo" o un "tramonto".

- La principale "sfera di riferimento", in cui ci si interrogava se l'immagine si riferisse ad un "contesto politico" o ad un "contesto privato". 


\section{Ocula}

Vol 21, No 22 (April 2020) • DOI: 10.12977/ocula2020-10

Marco Mazzoni e Roberto Mincigrucci | Il superleader ("ordinario"). Matteo Salvini

e il suo corpo

- Le attività condotte dal leader nelle immagini, che potevano riguardare diversi tipi di impegni lavorativi o attività legate alla propria sfera privata.

- L'abbigliamento, analizzando diverse scelte in termini di outfit, da quelle più formali a quelle più casual o informali.

\section{Il super celebrity politician: Salvini il "capitano"}

Nel suo profilo Facebook, Salvini si fa chiamare (e talvolta si autodefinisce) "il capitano". Questo appellativo è emblematico del tipo di immagine che l'ex vicepremier tenta di veicolare tramite la propria pagina pubblica. "Capitano" infatti è un termine molto noto soprattutto agli appassionati di sport: il capitano è uno dei giocatori di una squadra, uno che lotta insieme agli altri, sullo stesso terreno di gioco, per un obiettivo comune. Salvini non è il "presidente", che guarda i propri giocatori dalla tribuna, nemmeno "l'allenatore" che impartisce indicazioni agli altri stando seduto in panchina, Salvini è un giocatore che suda insieme agli altri mettendo la sua esperienza e le sue qualità al servi-

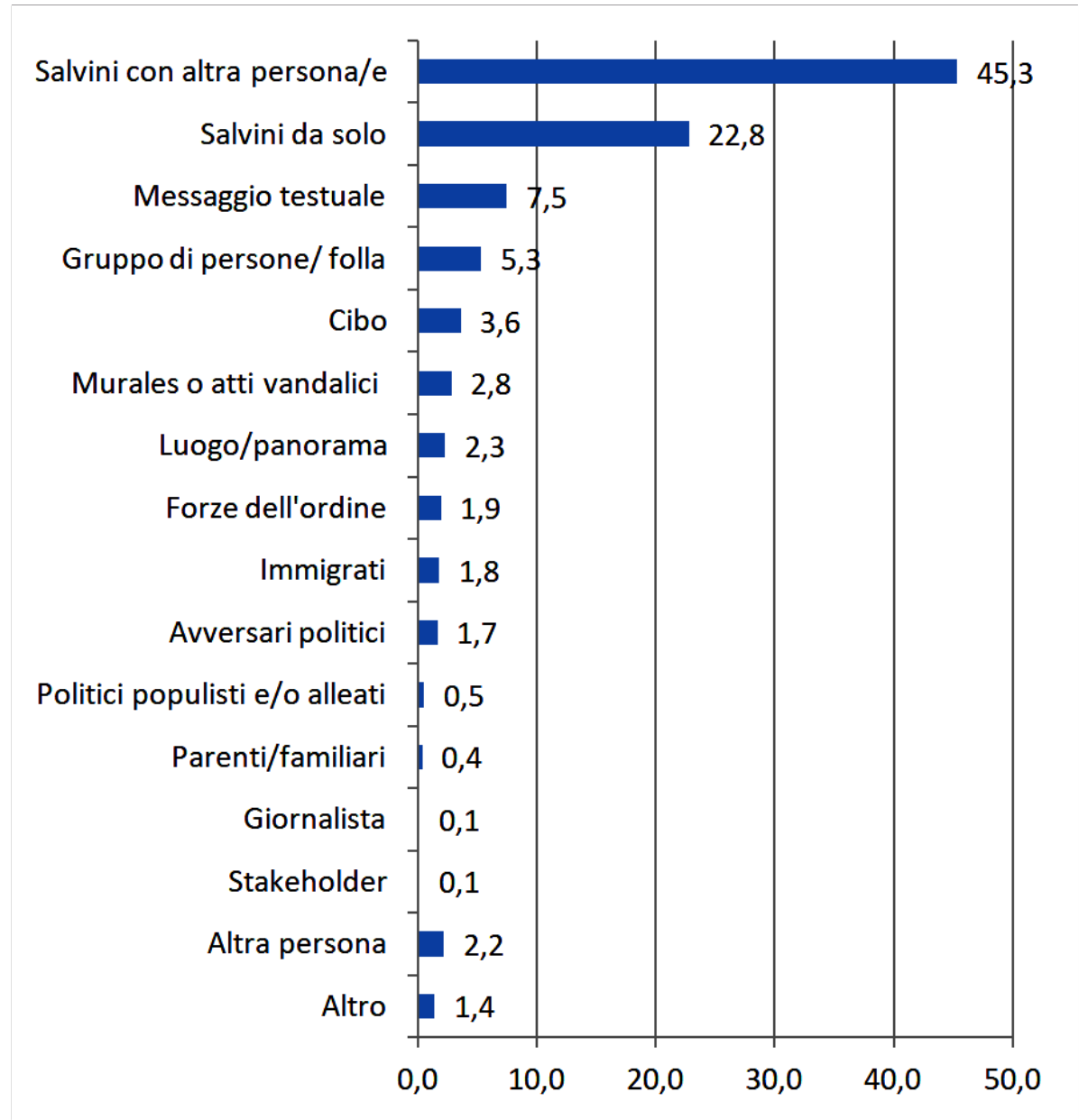

Figura 2. Soggetto principale dell'immagine (\%) (Tot. 772). 


\section{Ocula}

Vol 21, No 22 (April 2020) • DOI: 10.12977/ocula2020-10

Marco Mazzoni e Roberto Mincigrucci | Il superleader (“ordinario”). Matteo Salvini

e il suo corpo

zio della squadra. Il capitano però è anche il leader della squadra, il giocatore che si distingue dagli altri, che presenta delle caratteristiche superiori che gli permettono di guidare gli altri. Questo è il messaggio che Salvini cerca in ogni modo di trasmettere al proprio pubblico, mettendo a disposizione la propria immagine pubblica, e quindi il proprio corpo, per veicolarlo, come si può dedurre dai grafici che verranno mostrati di seguito.

La figura 2 indica il soggetto principale delle immagini analizzate. Il soggetto principale della foto poteva essere una persona fisica oppure un oggetto particolare, come ad esempio del "cibo" o un "tramonto". Quest'ultimi tipi di immagine verranno ripresi nel prossimo paragrafo. In questa sede ci limitano ad evidenziare l'alta tendenza a raffigurare Salvini insieme ad altre persone.

Come era prevedibile, Salvini è il protagonista della stragrande maggioranza delle immagini pubblicate sulla sua pagina. Tuttavia, nelle foto non appare come un leader solitario, ma condivide il suo protagonismo con altre persone (45,3 per cento delle immagini insieme ad altre persone contro 22,8 per cento delle immagini in cui è raffigurato da solo). Attraverso la scheda di analisi abbiamo cercato di andare più a fondo, creando una variabile che ci dicesse "da chi” è composto quel 45,3 per cento. Nella maggioranza relativa delle foto in cui Salvini compare con altri, il leader leghista si trova in mezzo ad un gruppo di persone, o meglio ad una folla (33 per cento dei casi²). Questo gruppo di persone può essere composto da militanti della Lega accorsi ad un comizio, manifestanti desiderosi di parlare con l'allora vicepremier per esprimere un disagio, membri di associazioni che lo invitano a delle feste e a delle iniziative. Tali immagini documentano i cosiddetti "bagni di folla", con Salvini che mette il proprio corpo a disposizione del suo "popolo", stringendo mani, ascoltando e prestandosi a dei selfie.

Le altre categorie di persone maggiormente raffigurate con Salvini sono le forze dell'ordine (27,7 per cento dei casi) o dei politici a lui vicini, leghisti o membri di partiti populisti europei (14,8 per cento). Questo dato conferma quanto abbiamo detto prima, ovvero che Salvini non agisce da solo, ma guida una comunità più ampia di persone. Inoltre, soprattutto l'alto tasso di presenza delle forze dell'ordine nelle sue foto, ci permette di introdurre un secondo aspetto che ci può far ricondurre il leader leghista ad un super celebrity politician: come detto in apertura di paragrafo, il capitano non è soltanto uno dei tanti membri di una squadra, ma è il membro che con le sue qualità superiori risolve i problemi della squadra. Salvini è un superleader, perché trasmette l'idea che è in grado di risolvere i problemi della gente comune. Tali problemi sono quelli tipici della cosiddetta "ideologia populista" (Mudde 2004) e perfettamente integrati nel complesso valoriale leghista (Turato 2018): le élite europee che vessano i lavoratori e i produttori italiani, la criminalità, gli immigrati. L’immagine principale che emerge dall'intera analisi è che Salvini è un leader che si impegna, lavora in maniera spasmodica e che dedica la sua vita all’impegno politico. Il suo corpo è continuamente esposto al servizio di que-

2 Questo dato si riferisce non alla totalità delle immagini analizzate ma soltanto a quelle in cui Salvini è immortalato insieme ad "altre persone" (350 foto in totale). 


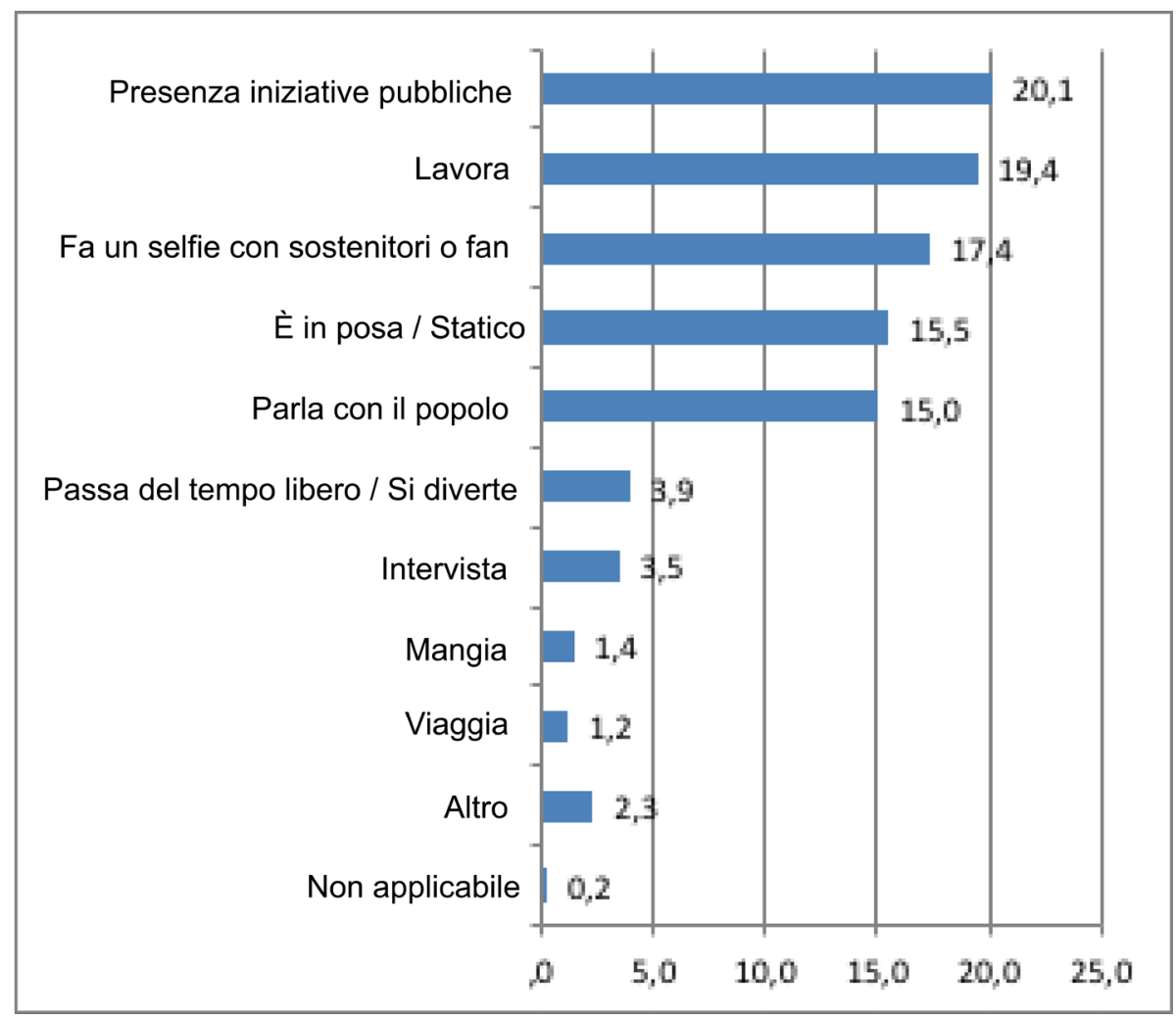

Figura 3. Che cosa fa Salvini nella foto? (\%) (Tot. 432).*

* Questo grafico non si riferisce a tutte le 772 immagini analizzate, ma soltanto a 432 in quanto sono state prese in considerazione soltanto le fotografie e i selfie che immortalavano Salvini.

sto messaggio, immortalato in contesti differenti e variegati, ma quasi sempre in funzione del suo compito: difendere le persone comuni dalle vessazioni delle élite e della globalizzazione.

Abbiamo cercato di comprendere il contesto di riferimento delle foto analizzate, dividendo le immagini relative alla sfera privata e personale da quelle che riguardano in qualche modo la sua attività politica. Sebbene questo risultato offra diversi spunti di riflessione, che verranno ripresi anche nel prossimo paragrafo, in questa sede ci limitiamo a dire che il risultato è piuttosto netto: l'88,2 per cento delle immagini analizzare riguarda il contesto politico, mentre il 9,8 per cento si riferisce alla sfera privata. ${ }^{3}$ Questo ha un significato ben preciso: nella sua pagina Facebook Salvini vuol far sapere alla gente che lavora. Lo stesso dato emerge in maniera ancora più chiara nella figura 3 , riferita al tipo di attività che stava conducendo Salvini nel momento in cui è stato immortalato. La categoria "lavora" si riferisce a quelle foto che ritraggono Salvini nel suo ufficio al ministero, intento ad esempio a firmare documenti, o mentre conduce meeting con i collaboratori o con degli interlocutori. La categoria "presenzia iniziative pubbliche" riguarda sempre l'ambito lavorativo,

3 L'1,9 per cento delle immagini non era codificabile secondo questa variabile. 


\section{Ocula $^{22}$}

Vol 21, No 22 (April 2020) • DOI: 10.12977/ocula2020-10

Marco Mazzoni e Roberto Mincigrucci | Il superleader ("ordinario"). Matteo Salvini

e il suo corpo

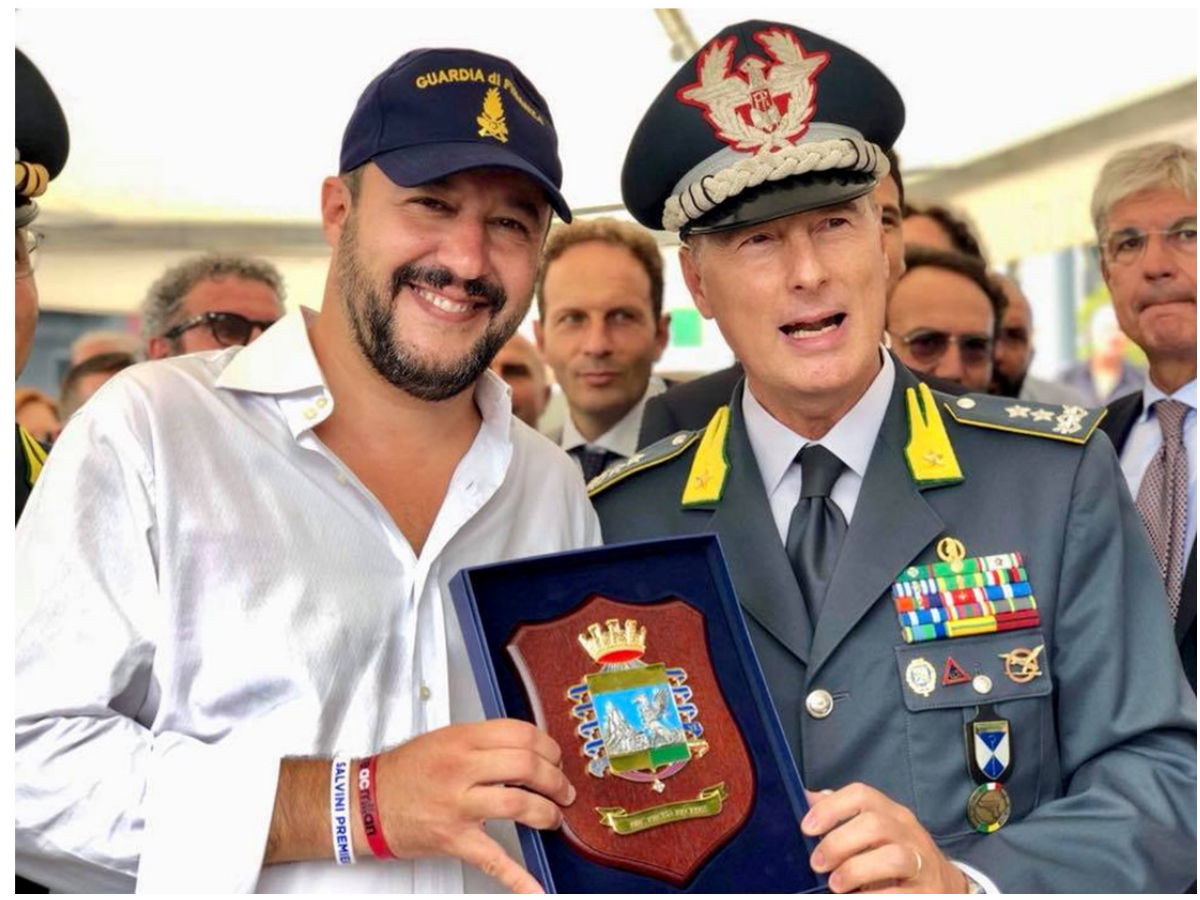

Figura 4. Foto pubblicata il 3/8/2018.

ma concerne un tipo di attività leggermente diversa: mentre parla ad un comizio, oppure partecipa ad appuntamenti istituzionali. La categoria "parla con il popolo" invece si riferisce a quelle situazioni in cui Salvini scende dal palco di un comizio per andare a parlare, ascoltare o stringere le mani alle persone che sono andate ad ascoltarlo, mentre saluta i suoi sostenitori.

Come si evince dalla figura 3, le due tipologie di attività maggiormente documentate nella sua pagina Facebook rimandano all'esercizio della sua attività politica: in alcuni casi seduto dietro ad una scrivania intento a "scrivere" il decreto sicurezza o a firmare i documenti per il rimpatrio di immigrati clandestini, in altre situazioni in mezzo alla gente, ad ascoltare i bisogni dei cittadini e a rassicurarli circa il suo operato.

Come abbiamo visto nei precedenti paragrafi, il corpo può essere considerato una "rappresentazione sociale intesa come nesso tra immagini mentali e icone corporee" (Pozzi 1994). Salvini utilizza nelle sue foto una vasta gamma di icone corporee che assumono un significato altamente simbolico e contribuiscono a far percepire l'impegno e la pragmaticità con cui affronta il suo compito. Le immagini che seguono sono un esempio di come il leader leghista utilizzi queste icone corporee per dimostrare di essere un superleader in grado di risolvere i problemi dei cittadini.

La figura 4 è un esempio tra le numerose immagini che lo vedono ritratto con degli esponenti delle forze dell'ordine. La massiccia presenza di militari nella sua pagina Facebook è essa stessa un simbolo: rimanda immediatamente ad uno dei temi cardine del programma leghista, ovvero quello della 


\section{Ocula $^{22}$}

Vol 21, No 22 (April 2020) • DOI: 10.12977/ocula2020-10

Marco Mazzoni e Roberto Mincigrucci | Il superleader (“ordinario”). Matteo Salvini

e il suo corpo

sicurezza. Mettendo il suo corpo in mezzo a quello di militari, carabinieri e finanzieri, Salvini lancia un messaggio ben preciso ai suoi followers, e cioè che l'ordine pubblico e la lotta alla criminalità sono per la sua attività politica delle vere e proprie priorità. Inoltre, come si evince dalla figura 4, Salvini fa qualcosa di più: indossa personalmente un simbolo delle forze dell'ordine, nel caso specifico il cappellino della Guardia di Finanza. Tra le immagini analizzate è piuttosto frequente vedere Salvini indossare un indumento delle forze dell'ordine, da un elmetto militare alle magliette della Polizia di Stato. Tali indumenti possono essere considerati delle icone che rimandano ad un messaggio ben preciso, ovvero che Salvini non considera soltanto il tema della sicurezza una priorità, ma che si impegna in prima persona alla sua tutela. Indossando il cappellino della Guardia di Finanza, l'allora vicepremier può far intendere che lui stesso può essere considerato, in qualche modo, un membro della Guardia di Finanza, e quindi che non è il classico politico che si limita a contrastare il crimine scrivendo leggi astratte, ma che è impegnato in prima linea, con atti concreti, a combattere i criminali.

Questo assunto appare ancora più evidente nella figura 5.

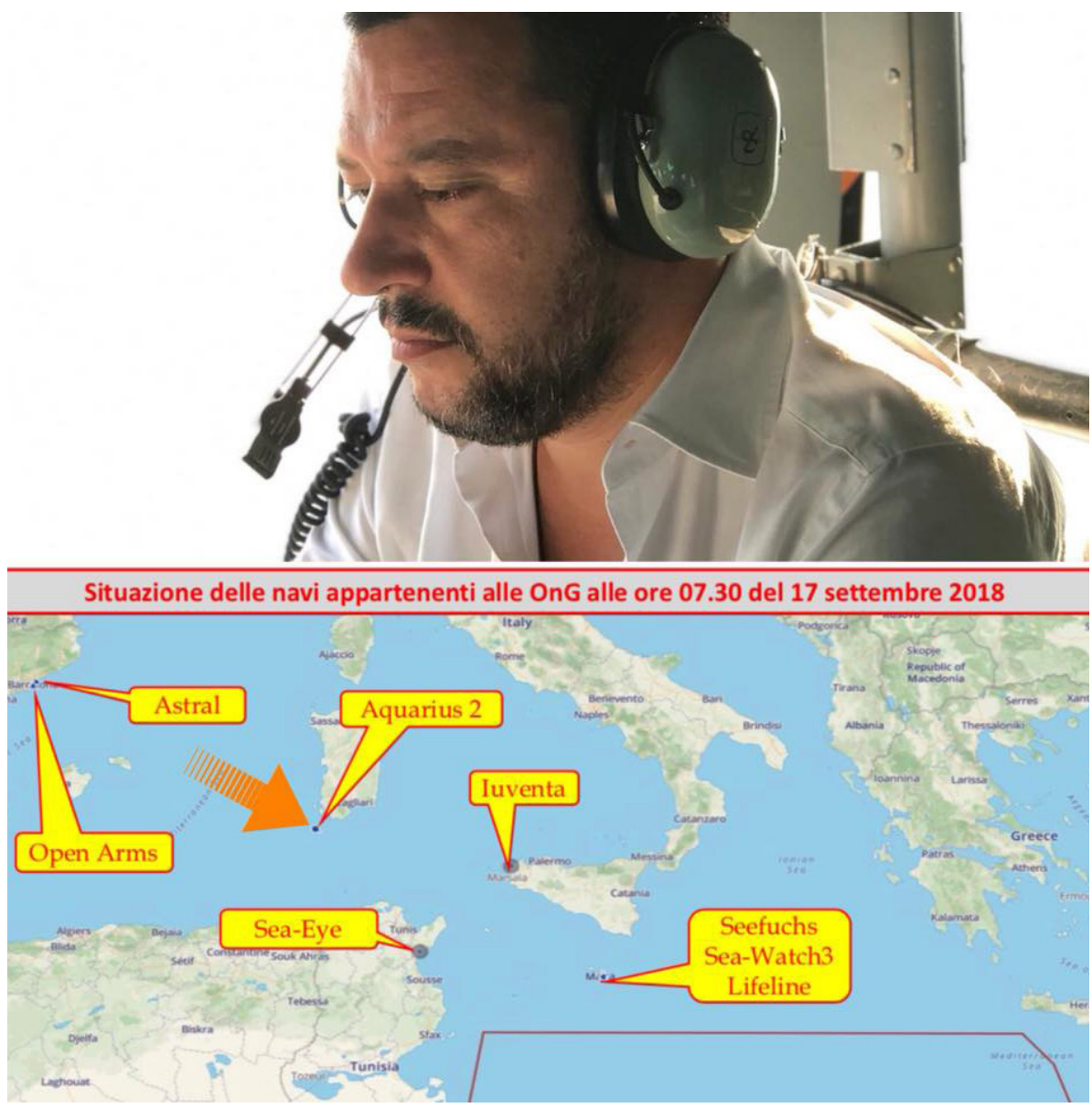

Figura 5. Pubblicata il 17/09/2018. 


\section{Ocula}

Vol 21, No 22 (April 2020) • DOI: 10.12977/ocula2020-10

Marco Mazzoni e Roberto Mincigrucci | Il superleader (“ordinario”). Matteo Salvini

e il suo corpo

Anche nella figura 5, come in quella precedente, Salvini indossa in prima persona un simbolo: le cuffie e il microfono dei marinai addetti al controllo dei radar. In questa immagine inoltre è allegata una cartina del Mar Mediterraneo con delle etichette riportanti le posizioni delle navi impegnate nel salvataggio dei migranti in mare. In questa foto il messaggio è abbastanza chiaro: Salvini non contrasta gli sbarchi e l'immigrazione clandestina limitandosi a scrivere decreti come di solito fanno i politici, ma è impegnato in prima persona a monitorare e a respingere le navi di quelle organizzazioni che disattendono le sue direttive. Il "superleader" diventa così un'icona pop, raffigurato come un supereroe dei fumetti intento a combattere, da sé, i nemici di turno, in questo caso coloro che tentano di far approdare in Italia dei migranti irregolari.

\section{L'everyday celebrity politicians - Salvini "l'ordinario"}

Nel precedente paragrafo abbiamo messo in luce quelle caratteristiche che riconducono Salvini alla figura del super celebrity politician teorizzato da Wood, Corbett e Flinders (2016): il leader leghista, nella sua pagina Facebook, appare spesso come un vero e proprio supereroe, che scende in strada a combattere il crimine e l'immigrazione clandestina. Tuttavia, già dai dati presentati nel paragrafo precedente si possono intravedere degli indizi che ci suggeriscono che Salvini non è soltanto "super", ma incarna anche alcune caratteristiche dell'everyday celebrity politician. Nonostante il suo costante impegno politico, Salvini non sale mai sul "piedistallo" istituzionale che lo porrebbe in una posizione sopraelevata rispetto ai suoi elettori. Salvini si pone sullo stesso piano del cittadino comune, cerca in continuazione il "bagno di folla". Ritornando alla figura 3 (presentata nel precedente paragrafo), nel 15 per cento delle foto analizzate il leader della Lega è intento a parlare con la sua gente, a salutare e stringere mani. Nel 17,4 per cento delle foto invece "presta il suo corpo" ai suoi sostenitori desiderosi di scattarsi un selfie. Attraverso il selfie, Salvini raffigura se stesso non come un membro di un'élite, separato dagli altri dalle transenne o dal cordone della security, ma come una persona normale, in grado di impersonare la quotidianità e lo stile di vita dei cittadini, innescando un processo di identificazione con l'elettorato (cfr. Ceccobelli 2017).

Nel paragrafo precedente abbiamo visto che i contesti in cui il leader leghista viene raffigurato nelle immagini sono perlopiù politici. Questo non vuol dire però che la sfera privata sia completamente assente dalla sua pagina Facebook. Come è stato molto discusso in letteratura (v. Stanyer 2012; Street 2004, 2012), la sfera privata di un leader può giocare un ruolo fondamentale nel creare empatia con il proprio elettorato, perché se il politico mette in mostra la sua personalità, e si presenta simile alla gente comune, lascia intendere che ha a cuore i suoi problemi e che quindi riesce a rappresentarla meglio di chiunque altro. Salvini probabilmente ne è consapevole e seppur in misura molto minore rispetto alle immagini relative alla sfera politica, pubblica quasi quotidianamente degli estratti di vita quotidiana che contribuiscono a renderlo più "umano" e "normale". Spesso questi estratti hanno cadenze orarie ben precise: la mattina prima di andare al lavoro, la sera tardi prima di andare a dormire. 


\section{Ocula $^{22}$}

Vol 21, No 22 (April 2020) • DOI: 10.12977/ocula2020-10

Marco Mazzoni e Roberto Mincigrucci | Il superleader ("ordinario"). Matteo Salvini e il suo corpo

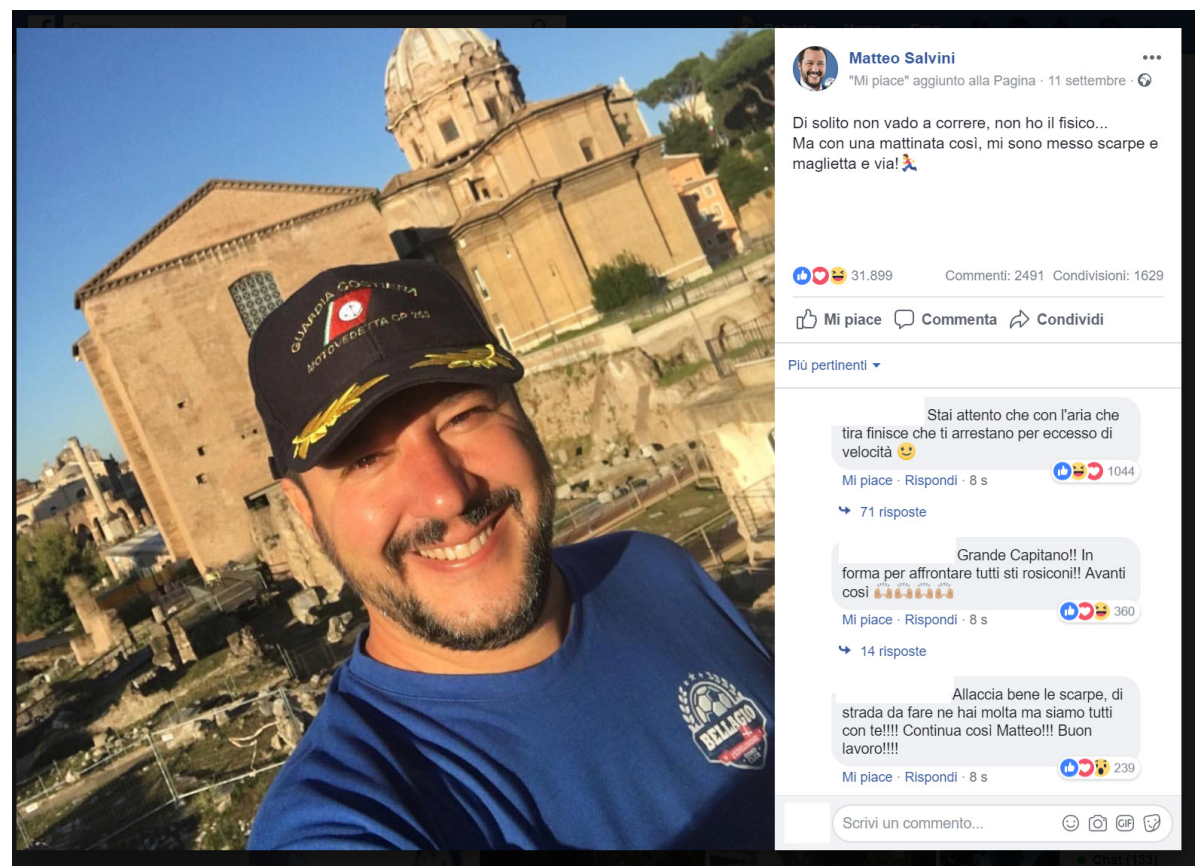

Figura 6. Foto pubblicata il 11/09/2018.

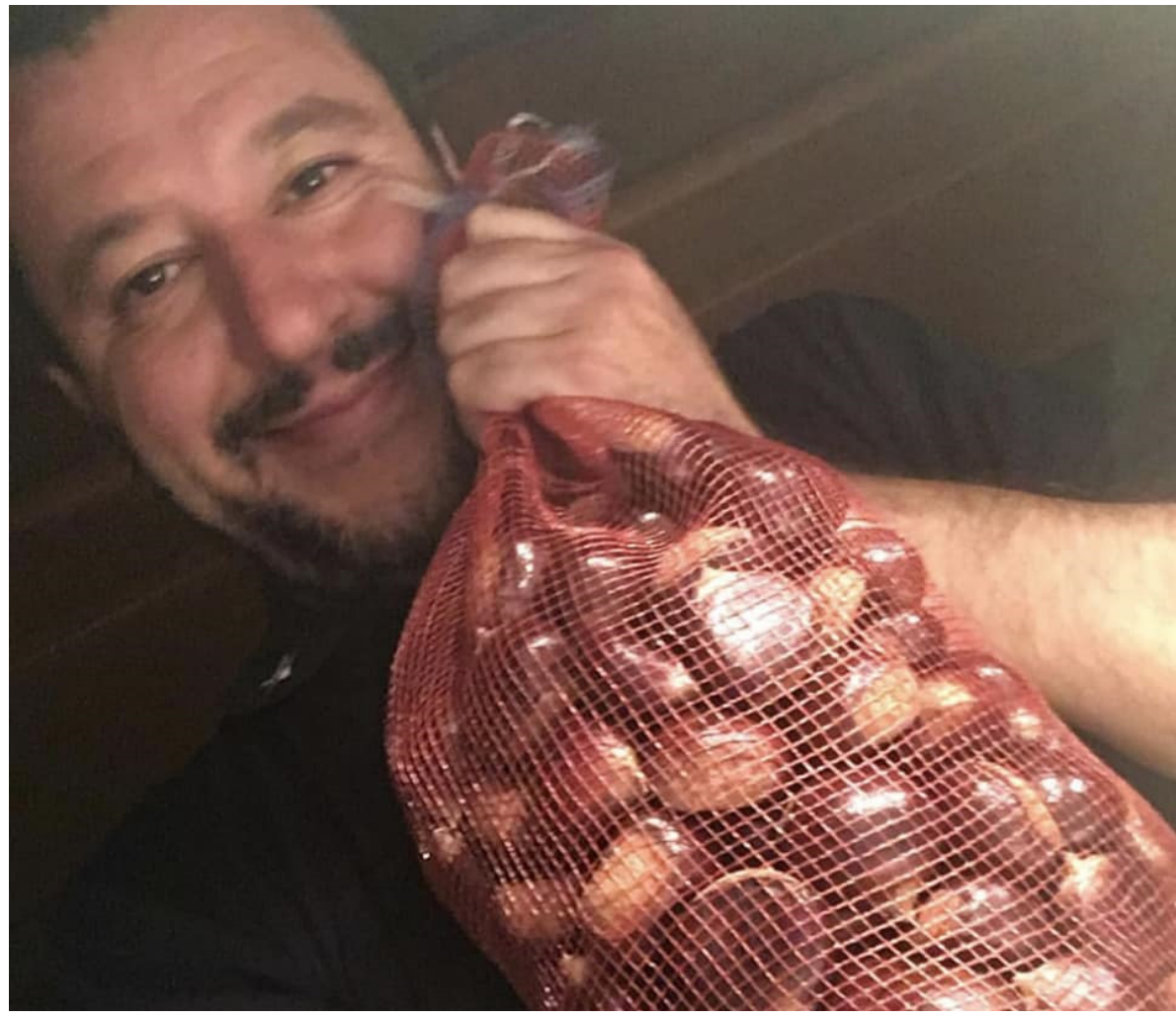

Figura 7. Foto pubblicata il 10/10/18. 


\section{Ocula $^{22}$}

Vol 21, No 22 (April 2020) • DOI: 10.12977/ocula2020-10

Marco Mazzoni e Roberto Mincigrucci | Il superleader ("ordinario"). Matteo Salvini

e il suo corpo

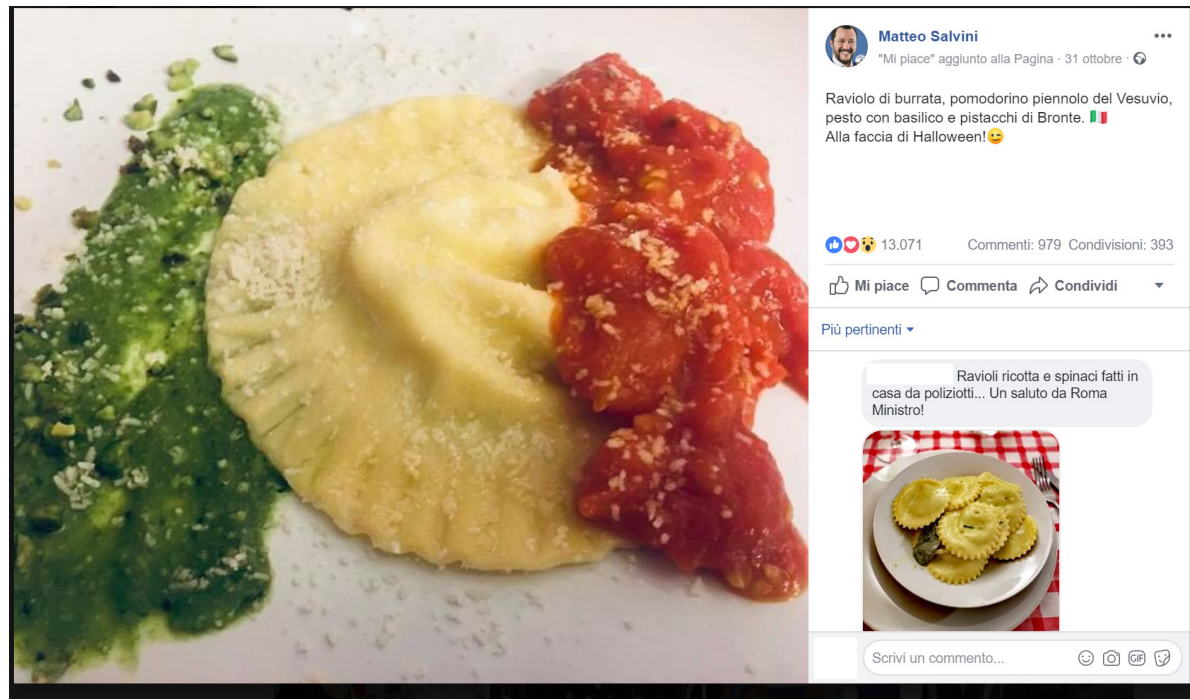

Figura 8. Foto pubblicata il 31/10/2018.

Prima di recarsi al lavoro pubblica le foto della corsetta mattutina, (indossando quasi sempre simboli delle forze dell'ordine, come la maglietta della polizia o il cappellino della guardia costiera [fig. 6]). La sera invece documenta prevalentemente momenti relax, come la "castagnata" mostrata in figura 7.

Nel precedente paragrafo abbiamo visto che Salvini dedica tantissimo spazio alla sua attività lavorativa, preoccupandosi di mostrarsi sempre attivo e impegnato a difendere gli interessi dei cittadini. Le figure 6 e 7 , come tante altre di quelle analizzate, dimostrano anche che Salvini è stato altrettanto bravo a variare la propria comunicazione, restituendo l'immagine di una persona "ordinaria" che dopo essere uscito dall'ufficio chiude la cartellina dei documenti e si concede dei momenti di relax, dalle castagne e vino rosso alla corsetta mattutina, passando per la partita allo stadio la domenica sera, esattamente come fa la maggior parte delle persone comuni. Da queste immagini si può notare chiaramente la "doppia veste" di Salvini, leader super impegnato, deciso e talvolta duro in politica, ma persona semplice e rilassata a casa, come qualsiasi persona che cerca di conciliare gli impegni di lavoro con la famiglia.

Un ruolo importante, nella costruzione dell'immagine del "Salvini casalingo", lo riveste il cibo. Riprendendo la figura 2 presentata del precedente paragrafo, il 3,6 per cento delle immagini analizzate riguarda il cibo. Fotografare il piatto prima di mangiarlo, e condividerlo con i propri followers sul proprio profilo, è una pratica che sta diventando sempre più diffusa tra gli utenti dei social network. Salvini non si sottrae a questa moda, e come i cittadini comuni anche lui condivide con i suoi fan le portate dei suoi pasti. L'unica condizione è "l'italianità" dell'alimento. Nel profilo di Salvini non compaiono mai sushi, tapas o formaggio francese, ma soltanto ricette della tradizione italiana (fig. 8), in piena coerenza con il suo messaggio politico. Come sostiene Bennet (1998), le scelte di consumo e il tenore di vita assumono una valenza politica e un leader politico non deve essere solo il fautore di una determinata proposta poli- 


\section{Ocula}

Vol 21, No 22 (April 2020) • DOI: 10.12977/ocula2020-10

Marco Mazzoni e Roberto Mincigrucci | Il superleader ("ordinario"). Matteo Salvini

e il suo corpo

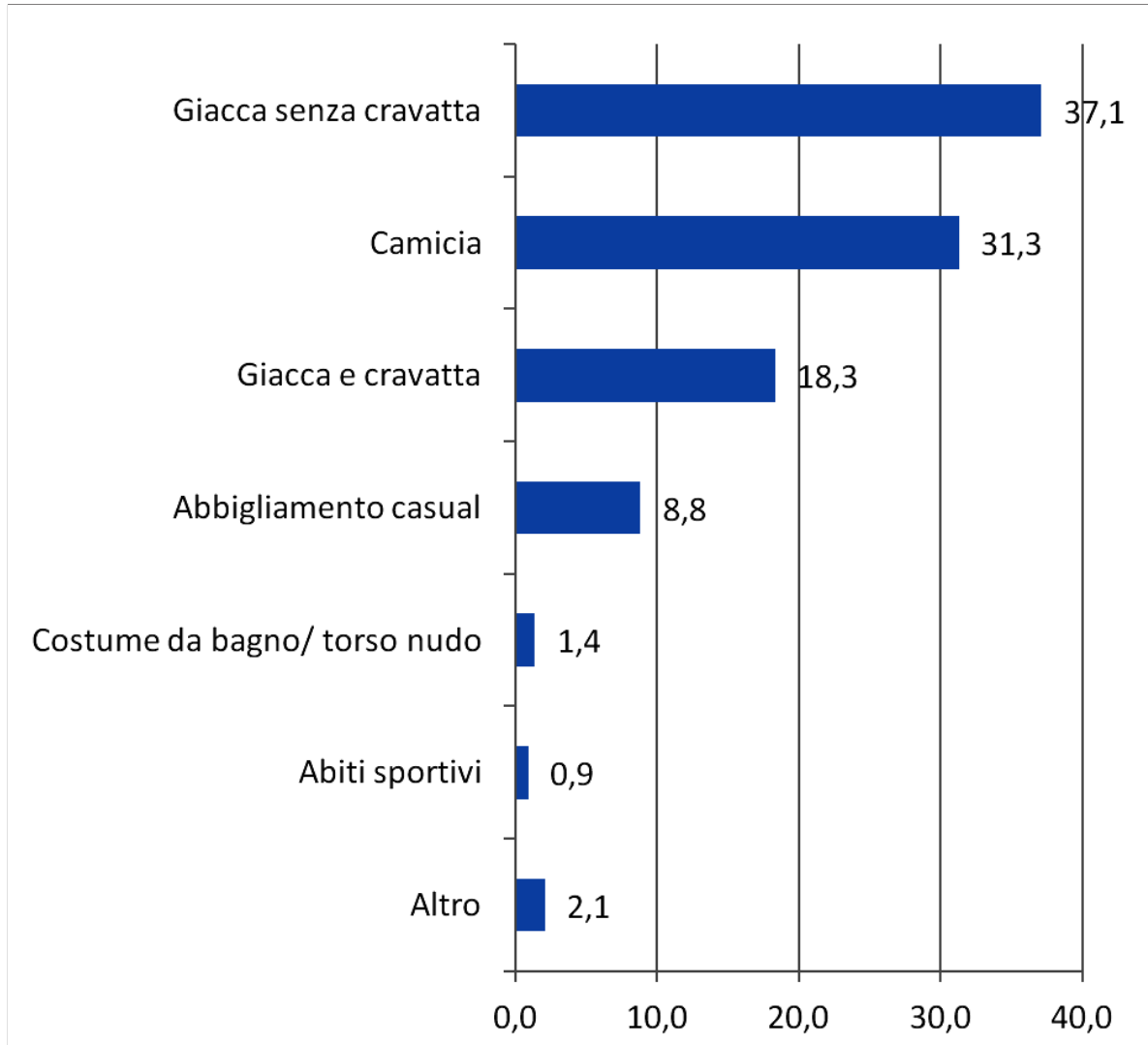

Figura 9. Abbigliamento di Salvini nelle foto (\%) (Tot. $432^{* *}$ ).

** Questo grafico non si riferisce a tutte le 772 immagini analizzate, ma soltanto a 432 in quanto sono state prese in considerazione soltanto le fotografie e i selfie che immortalavano Salvini.

tica, ma deve essere coerente con il gruppo sociale che intende rappresentare, con una piena adesione al modello di vita che vuole identificare.

Come il cibo, anche il tipo di abbigliamento può essere considerato un simbolo in grado di veicolare un messaggio ben preciso al proprio elettorato. La figura 9 si riferisce al tipo di abbigliamento mostrato da Salvini nelle sue foto della pagina.

La figura 9 lo dimostra in maniera abbastanza chiara: la classica divisa di un membro della classe dirigente, ovvero il vestito giacca e cravatta, sono utilizzati lo stretto indispensabile dall'ex Ministro degli Interni (18,3 per cento dei casi): soltanto in appuntamenti particolarmente formali, dove è presente anche il Presidente Mattarella, o in importanti meeting internazionali. Il "corpo" di Salvini è, nella maggior parte dei casi, raffigurato senza cravatta e nel 31,3 per cento dei casi anche senza giacca, con una semplice camicia. Salvini è un leader che spinge all'estremo la messa in scena del proprio corpo come strumento di identificazione con il proprio elettorato e l'1,4 per cento di immagini che lo ritraggono in costume da bagno, senza maglietta, ne sono un esempio. Il 10 agosto Salvini pubblica una foto (fig. 10) in cui è immortalato a 


\section{Ocula $^{22}$}

Vol 21, No 22 (April 2020) • DOI: 10.12977/ocula2020-10

Marco Mazzoni e Roberto Mincigrucci | Il superleader ("ordinario"). Matteo Salvini

e il suo corpo

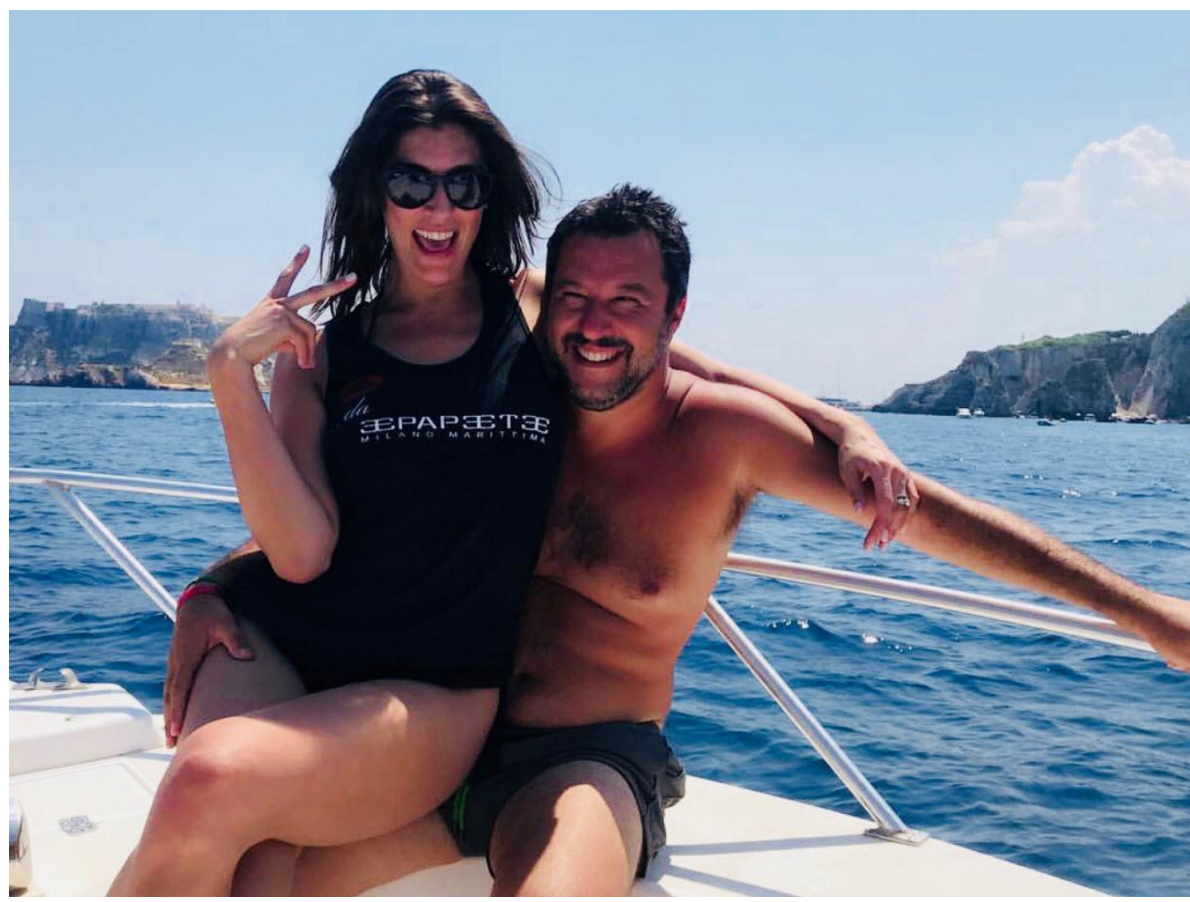

Figura 10. Foto pubblicata il 31/10/2018.

"torso nudo", con la sua ex fidanzata, Elisa Isoardi, seduta sulle sue ginocchia sfoggiando la canottiera di una nota discoteca della riviera romagnola. Una scelta comunicativa profondamente differente rispetto a quelle adottate dai politici del passato, quando Aldo Moro si faceva ritrarre in giacca e cravatta anche sulla spiaggia, perché in quanto rappresentante delle istituzioni doveva sempre esibire un abbigliamento formale.

Non è molto frequente vedere dei ministri diffondere volontariamente delle proprie immagini a torso nudo, e tale scelta ha suscitato più di una perplessità da parte di alcuni giornalisti ed osservatori. A nostro parere però queste immagini non sono una boutade estiva, non rappresentano un uso superficiale dei social, ma dimostrano una scelta consapevole finalizzata a renderlo allo stesso tempo più "celebre" ma anche più "normale", più simile all'italiano medio. In queste fotografie Salvini, più che rappresentare le istituzioni, probabilmente intende identificarsi con il proprio elettorato, un tipo di persone che difficilmente veste abiti formali e che ad agosto riempie la propria bacheca Facebook con le foto delle vacanze.

Per riassumere, quando comunica la sua attività politica, Salvini tende a mostrarsi come un "superleader", pragmatico e decisionista, che non ha paura di alzare la voce per portare avanti le sue battaglie populiste. Può essere considerato a tutti gli effetti un leader forte (cfr. Calise 2018: 237), ma quando si parla della sua sfera privata l'immagine del superleader scompare, ne emerge un personaggio "ordinario", perfettamente integrato con quei "perdenti della globalizzazione" che stanno alla base del suo consenso. 


\section{Ocula}

Vol 21, No 22 (April 2020) • DOI: 10.12977/ocula2020-10

Marco Mazzoni e Roberto Mincigrucci | Il superleader (“ordinario”). Matteo Salvini

e il suo corpo

\section{Conclusioni}

Attraverso questo studio abbiamo cercato di dimostrare che Salvini è sempre di più il "superleader" (Boni 2008) della politica contemporanea. Non è soltanto un politico che detta l'agenda con le sue proposte programmatiche, ma una vera e propria "icona pop" (Spaziante 2016), un personaggio glamour, perfettamente integrato con i canoni della cultura di massa e che attira l'attenzione anche per lo "stile" con cui caratterizza sia il suo agire politico che le sue abitudini private. La popolarità di cui gode il leader leghista passa soprattutto per una sapiente rappresentazione del suo corpo, associato ad una vasta gamma di simboli e veicolato attraverso una costante diffusione di immagini nella sua pagina Facebook. In particolare Salvini, nella rappresentazione mediatica del suo corpo, indossa una "duplice veste": quella di super celebrity politician quando si occupa di politica ma anche everyday celebrity politician quando mette in scena elementi della sua vita privata o alcuni dettagli del suo abbigliamento e delle sue scelte di consumo.

Il risultato che possiamo evidenziare da quanto fin qui detto è che nell'epoca contraddistinta dal populismo e dall'antipolitica, la "messa in scena del retroscena" e la conseguente "desacralizzazione" teorizzata da Meyrowitz è una strategia efficace per i leader per costruire un rapporto di fiducia con il proprio elettorato. Inizialmente Street (2004) ha rilevato che il politico raggiunge lo status di celebrità grazie soprattutto alle sue qualità eccezionali: qualità riconosciute dai cittadini e ritenute necessarie per governare un paese. Conclusione da cui si allontanano, come già accennato, Wood, Corbett e Flinders (2016) sostenendo invece che la celebrità del leader politico serve per mostrarsi nel modo più autentico e normale possibile per il semplice fatto che il cittadino desidera governanti che gli assomiglino. L'analisi del "corpo mediale" di Salvini ci permette di giungere a un risultato un po' più articolato. La nostra analisi, infatti, ci dice che Salvini riesce a mixare le qualità del "superleader" con quelle della persona "ordinaria". Nel periodo in cui viviamo i leader sono chiamati ad affrontare problematiche sempre più complesse che richiedono specifiche qualità, ma al contempo la gente preferisce affidarsi a governanti che presentano tratti simili alle persone comuni. Come abbiamo detto prima, Salvini si è rivolto prevalentemente ai cosiddetti "perdenti della globalizzazione", persone sfiduciate dalla politica e che avvertono le difficoltà derivanti della crisi economica. Un tipo di elettorato che difficilmente entrerebbe in empatia con un leader che si presenta esclusivamente come una "super celebrity" o "superleader".

Salvini è riuscito a nostro parere in questo mix: ha diffuso un'immagine di sé ben precisa, una icona pop con le qualità giuste per risolvere i molti problemi che affliggono il paese ma anche un "uomo comune", una persona simile e vicina agli individui a cui si rivolge. 


\section{Ocula}

Vol 21, No 22 (April 2020) • DOI: 10.12977/ocula2020-10

Marco Mazzoni e Roberto Mincigrucci | Il superleader ("ordinario"). Matteo Salvini

e il suo corpo

\section{Bibliografia}

Achen, Christopher H.; Bartels, Larry .M.

2016 Democracy for Realists: Why Elections do not Produce Responsive Government, Princeton, University Press.

Bartlett, Jamie

2014 "Populism, social media and democratic strain", in Sandelind, C. (ed.), European Populism and Winning the Immigration Debate, Falun, ScandBook.

Bennet, Lance

1998 "The uncivic culture: Communication, identity and the rise of lifestyle politics", Political Science e Politics, 31, 4, 741-761.

Bobba, Giuliano

2019 "Social media populism: Features and 'likeability'of Lega Nord communication on Facebook", European Political Science, 18, 1, 11-23.

Boni, Federico

2008 Il Superleader. Fenomenologia mediatica di Silvio Berlusconi, Roma, Meltemi.

Boni, Federico

2002 Il corpo mediale del leader. Rituali del potere e sacralità del corpo nell'epoca della comunicazione globale, Roma, Meltemi.

Brile, Nello; Vagni, Tito

2019 "Lo-fi politics. Il brand del politico e lo stile amatoriale in bassa fedeltà". Comunicazione politica, 1, 2019, 63-84.

Calise, Mauro

2018 "Partiti e leader: istruzioni per l'uso", in Valbruzzi, M. e Vignati, R. (a cura di), Il Vicolo Cieco. Le elezioni del 4 marzo 2018, Bologna, Il Mulino.

Carone, Martina; Cavallaro, Matteo

2019 "Salvini social”, in Diamanti, G.; Pregliasco, L. (a cura di), Fenomeno Salvini. Chi è, come comunica, perché lo votano, Roma, Castelvecchi.

Ceccobelli, Diego

2017 Facebook al potere. Lo stile della leadership al tempo dei social media, Santarcangelo di Romagna, Maggioli.

Delli Carpini, Michael X.; Williams, Bruce A.

2001 "Let us infotain you: Politics in the new media age", in Bennett W. L .e Entman, R. L. (ed.), Mediated politics: Communication in the future of democracy , Cambridge - New York, Cambridge University.

De Vreese, Claes H. et al.

2018 "Populism as an Expression of Political Communication Content and Style: A New Perspective”, The International Journal of Press/Politics, 23, 4, 1-16.

Diamanti, Giovanni

2019 "La nuova Lega: un rebranding che parte da lontano", in Diamanti, G. e Pregliasco, L. (a cura di), Fenomeno Salvini. Chi è, come comunica, perché lo votano, Roma, Castelvecchi. 


\section{Ocula}

Vol 21, No 22 (April 2020) • DOI: 10.12977/ocula2020-10

Marco Mazzoni e Roberto Mincigrucci | Il superleader ("ordinario"). Matteo Salvini

e il suo corpo

Engesser, Sven; Fawzi, Nayla; Larsson, Anders Olof

2017 "Populist Online Communication: Introduction to the Special Issue", Information, Communication \& Society, 20, 9, 1279-1292.

Grabe, Maria Elizabeth; Bucy, Erik Page

2009 Image bite politics: News and the visual framing of elections. Oxford, OxfordUniversity Press.

Green, Donald P.; Palmquist, Bradley; Schickler, Eric

2002 Partisan Hearts and Minds: Political Parties and the Social Identities of Voters, New Haven,Yale University Press.

Kane, John; Patapan, Haig

2012 The Democratic Leader: How Democracy Defines, Empowers and Limits Its Leaders, Oxford, Oxford University Press.

Karadimitriou, Achilleas; Veneti, Anastasia

2016 "Political Selfies: Image Events in the New Media Field", in Karatzogianni, A.; Nguyen, D.; Serafinelli, E. (eds.), Digital Transformation of the Public Sphere. London, Palgrave Macmillan.

Kreiss, Daniel

2018 "The Media Are About Identity, Not Information", in Boczkowski, P. e Papacharissi, Z. (eds.), Trump and the Media, Cambridge-London, MIT Press.

Kriesi, Hanspeter

2014 “The populist challenge”, West European Politics, 37, 2, 361-378.

Kriesi, Hanspeter et al.

2006 "Globalization and the Transformation of the National Political Space: Six European Countries Compared”, Europan Journal of Political Research, 45, 6, 921-956.

Mancini, Paolo

2011 Between Commodification and Lyfestyle Politics: Does Silvio Berlusconi Provide a New Model of Politics for the Twenty-first Century?, Oxford, Reuters Institute.

Marsh, David; Hart, Paul T.; Tindall, Karen.

2010 "Celebrity politics: The politics of the late modernity?", Political Studies Review, 8, 3, 322-340.

Mazzoleni, Giampietro; Bracciale, Roberta

2019 La politica pop online. I meme e le sfide della comunicazione politica, Bologna, Il Mulino.

Mazzoni, Marco; Ciaglia, Antonio

2015 Il gossip al potere. Il politico celebrità nell'era della politica pop, Sant'Arcangelo di Romagna, Maggioli.

Meyrowitz, Joshua

1985 No sense of place: the impact of electronic media on social behavior, Oxford, Oxford Univesity Press. 


\section{Ocula}

Vol 21, No 22 (April 2020) • DOI: 10.12977/ocula2020-10

Marco Mazzoni e Roberto Mincigrucci | Il superleader ("ordinario"). Matteo Salvini

e il suo corpo

Mudde, Cas

2004 "The populist zeitgeist”, Government e Opposition, 39, 4, 542-563.

Muñoz, Caroline Lego; Towner, Terri L.

2017 "The image is the message: Instagram marketing and the 2016 presidential primary season”, Journal of Political Marketing, 16, 3-4, 290-318.

Passarelli, Gianluca; Tuorto, Dario

2018 "La Lega dopo il voto del 4 marzo", Rivista Il Mulino, 2, 265-271.

Pozzi, Enrico

1994 "Per una sociologia del corpo", Il corpo II, 2, 106-144.

Schill, Dan

2012 "The visual image and the political image: A review of visual communication research in the field of political communication", Review of Communication, 12,2 , p 118-142.

Spaziante, Lucio

2016 Icone pop. Identità e apparenze tra semiotica e musica, Milano, Bruno Mondadori.

Stanyer, James

2012 Intimate Politics: Publicity, Privacy and Personal Lives of Politicians in Media-Saturated Democracies, Cambridge, Polity Press.

Street, John

2004 "Celebrity politicians: popular culture and political representation", The British Journal of Politics and International Relations, 6, 4, 435-452.

2012 "Do celebrity politics and celebrity politicians matter?", The British Journal of Politics and International Relations, 14, 3, 346-356.

2019 "What is Donald Trump? Forms of 'Celebrity' in Celebrity Politics", Political Studies Review, 17, 1, 3-13.

Turato, Fabio

2018 Capipopolo. Leader e leadership del populismo europeo, Roma, Castelvecchi.

Valbruzzi, Marco; Vignati, Rinaldo

2018 Il Vicolo Cieco. Le elezioni del 4 marzo 2018, Bologna, Il Mulino.

Vampa, Davide

2018 "Il Centro destra a guida leghista", in Valbruzzi, M. e Vignati, R. (a cura di), Il Vicolo Cieco. Le elezioni del 4 marzo 2018, Bologna, Il Mulino.

Van Zoonen, Liesbet

2005 Entertaining the Citizen: When Politics and Popular Culture Converge, Lanham, MD, Rowman \& Littlefield.

Wheeler, Mark

2013 Celebrity Politics, Cambridge, Polity Press.

Wood, Matthew; Corbett, Jack; Flinders, Matthew

2016 "Just like us: Everyday celebrity politicians and the pursuit of popularity in an age of anti-politics", The British Journal of Politics and International Relations, 18, 3, 581-598. 


\section{Ocula ${ }^{22}$}

Vol 21, No 22 (April 2020) • DOI: 10.12977/ocula2020-10

Marco Mazzoni e Roberto Mincigrucci | Il superleader ("ordinario”). Matteo Salvini

e il suo corpo

Marco Mazzoni è Professore associato in Sociologia dei Processi Culturali e Comunicativi presso il Dipartimento di Scienze Politiche dell’Università degli Studi di Perugia. Insegna Lobbying e Relazioni Pubbliche e Sistemi Politici e Tecniche di Comunicazione alla magistrale di Comunicazione Pubblica, Digitale e d'Impresa. Suoi articoli sono apparsi su "Journalism”, “Journalism Studies", "The International Journal of Press/ Politics", "European Journal of Communication", "International Journal of Cultural Studies”.

Roberto Mincigrucci è Assegnista di ricerca in Sociologia dei Processi Culturali e Comunicativi presso il Dipartimento di Scienze Politiche dell'Università degli Studi di Perugia. Collabora con la cattedra di Lobbying e Relazioni Pubbliche e Introduzione al Sistema Italiano dei Media presso lo stesso dipartimento. I principali temi di ricerca riguardano la corruzione, gli studi sul giornalismo e la comunicazione politica. In particolare, negli ultimi anni ha condotto alcuni studi sulla copertura giornalistica del tema della corruzione e sul ruolo dei quotidiani nelle recenti campagne elettorali. Recentemente sta lavorando ad una ricerca sull'uso delle immagini nei social network da parte di alcuni attori politici. 